Théologiques

Théologiques

\title{
Dialoguer, encore et toujours, avec les musulmans
}

\section{Maurice Borrmans}

Volume 19, numéro 2, 2011

Le dialogue islamo-chrétien

URI : https://id.erudit.org/iderudit/1024726ar

DOI : https://doi.org/10.7202/1024726ar

Aller au sommaire du numéro

\section{Éditeur(s)}

Faculté de théologie et de sciences des religions, Université de Montréal

\section{ISSN}

1188-7109 (imprimé)

1492-1413 (numérique)

Découvrir la revue

\section{Citer cet article}

Borrmans, M. (2011). Dialoguer, encore et toujours, avec les musulmans. Théologiques, 19(2), 17-40. https://doi.org/10.7202/1024726ar

\section{Résumé de l'article}

Les Églises locales, en Europe, ont multiplié leurs institutions pour dialoguer avec les musulmans immigrés pour en développer toutes les possibilités : à côté de leurs fidèles engagés dans ce dialogue, beaucoup d'autres le critiquent ou le refusent pour mille raisons politiques ou culturelles, voire théologiques et spirituelles. Les papes Jean Paul II et Benoît XVI insistent sur la nécessité de le poursuivre « encore et toujours » au nom même de l'Évangile, malgré les difficultés du moment. Même l'incident de parcours que fut la conférence de Ratisbonne (12 septembre 2006) s'est révélé être une incitation à en approfondir les modalités et les limites. Quant à la Lettre des 138 personnalités musulmanes d'octobre 2007, riche en promesses, elle a été trop vite oubliée de part et d'autre. La Lettre du Cardinal Martini au peuple de Milan, de décembre 1990, est pourtant toujours d'actualité. Les chrétiens sont invités à rendre compte de leur foi, à bien connaître les musulmans et à leur faire découvrir les valeurs chrétiennes de la culture européenne. Tous doivent être réalistes dans leurs collaborations en y tenant compte des difficultés qu'il y a à " vivre ensemble " quand on appartient à des cultures différenciées : que chacun y fasse effort pour être plus proche de l'autre. 


\section{Dialoguer, encore et toujours, avec les musulmans"}

Maurice BorRmans**

Chrétiens et musulmans peuvent-ils encore dialoguer alors que beaucoup craignent un "choc des civilisations" et que nombreux sont ceux et celles qui prônent un «repli identitaire» ? Le fait est qu'en Europe occidentale, les exigences de la laïcité, d'une part, et la volonté de visibilité des musulmans, d'autre part, engendrent bien des problèmes dans les opinions publiques et les communautés chrétiennes. D’autant plus que, dans les pays dits islamiques, les «printemps arabes» voient l'émergence politique de mouvements fondamentalistes ou même salafistes qui ne sont pas sans créer également de nouveaux obstacles au «vivre ensemble» auquel s'étaient habitués les chrétiens du Moyen-Orient ou du Maghreb. Si, dans les autres pays européens, les immigrations se sont stabilisées, il n'empêche que, partout, les musulmans s'y sont organisés en associations et fédérations et leur spécificité s'affirme toujours plus dans l'espace public. Si les

* Le présent article constitue une version remaniée de celui qui a été publié initialement en italien dans la revue $A d$ Gentes, ce qui explique le développement qui propose un regard rétrospectif sur la lettre de $\mathrm{M}^{\mathrm{gr}}$ Martini (section 4), par ailleurs peu connue dans les milieux francophones. Ad Gentes se trouve donc à avoir publié trois numéros thématiques sur le dialogue islamo-chrétien: Cristiani e musulmani in Europa, Ad Gentes, 9/2 (2005), Cristiani e musulmani nel mondo, Ad Gentes, 11/2 (2007) et Cristiani e musulmani oggi in Italia, Ad Gentes, 17/1 (2013).

* Le Père Maurice Borrmans, membre de la Société des Missionnaires d'Afrique (Pères Blancs), est professeur retraité du Pontificio Istituto di Studi Arabi e d'Islamistica (PISAI) de Rome. Spécialiste du droit islamique, de la spiritualité musulmane et de l'histoire des rapports islamo-chrétiens, il a longtemps été consultant auprès du Conseil Pontifical pour le Dialogue Interreligieux. Parmi ses nombreuses publications, mentionnons: (2009) Prophètes du dialogue islamo-chrétien: Louis Massignon, Jean-Mohammed Abd-el-Jalil, Louis Gardet, Georges C. Anawati, Paris, Cerf; (2009) Mulla-Zadé et Abd-el-Jalil, Deux frères en conversion, du Coran à Jésus (correspondance 1927-1957), rassemblée, introduite et annotée par M. Borrmans, Paris, Cerf; (2011) Dialoguer avec les Musulmans: une cause perdue ou une cause à gagner?, Paris, Téqui.

C Revue Théologiques 2011. Tout droit réservé. 
Églises européennes ont multiplié leurs institutions de dialogue et s'efforcent de développer celui-ci sous toutes ses formes, elles voient cependant un nombre grandissant de leurs fidèles critiquer, contester et même refuser tout dialogue interreligieux pour de nombreux prétextes qui vont des plus politiques et culturels au plus théologiques et spirituels. Certains n'hésitent pas à poser la question: "À quoi le dialogue islamo-chrétien a-t-il servi depuis cinquante ans?» D'autres s'interrogent alors: "Ne s'agirait-il pas d'une "cause perdue" ? Et beaucoup de déclarer inéluctable un «choc des civilisations ", comme le suggérait Samuel P. Huntington, il y a quelques années. Certains préfèrent y voir un «choc des ignorances» réciproques, et ils ont raison, bien qu'un grand nombre de facteurs nouveaux soient venus compliquer les rapports interreligieux au cours des dernières décennies. Le dialogue islamo-chrétien serait-il en crise et faudrait-il "changer de cap ", comme le suggère un livre récemment publié en France ${ }^{1}$ ? Voilà pourquoi il nous faut bien mesurer quelles sont «les chances et les limites » de ce dialogue, dans les conditions actuelles, au-delà des polémiques ou des malentendus. C'est pourquoi, après avoir publié, en France, Dialogue islamo-chrétien à temps et contretemps, (Borrmans 2002), j'ai cru devoir récidiver avec un livre dont le titre s'est voulu provocateur: Dialoguer avec les musulmans: une cause perdue ou une cause à gagner? (Borrmans 2012).

Dans le cadre de ce dossier de la revue Théologiques, j'entends donc prendre la mesure des défis du dialogue, par un retour réflexif qui retrace surtout le parcours de quelques interventions magistérielles. Notre balise temporelle ne sera pas tant celle du 11 septembre 2001 que la conférence de Benoît XVI à Ratisbonne, qui a paradoxalement rappelé à tous combien ce dialogue s'avère plus que jamais nécessaire et urgent (section 2). Or, les textes du concile Vatican II, ceux des derniers papes et du Conseil pontifical pour le dialogue interreligieux - qui ne cessent d'insister sur la nécessité du dialogue - sont toujours valables, même si certains semblent aujourd'hui les oublier et mettent en cause le bienfondé des initiatives prises du côté chrétien comme du côté musulman, depuis plus de quarante ans (section 1). La Lettre des 138 personnalités musulmanes d'octobre

1. Voir Gallez (2012) pour qui «le dialogue islamo-chrétien est en crise » parce que ses protagonistes se sont trompés dans «l'élaboration du concept du "non-chrétien" ", parce que la théologie latine s'est trompée quant au «salut personnel et son présupposé théologique », parce qu'on y a suivi «la gnose mysticisante» de L. Massignon et parce qu'on n'a pas voulu reprendre l'étude des origines de l'islam, selon les méthodes historico-critiques. 
2007 répondait en quelque sorte, de manière constructive, à l'intervention de Ratisbonne, mais elle était adressée à tous les chefs de communautés chrétiennes du monde entier (section 3). Elle pouvait être considérée comme un signe d'espérance, mais elle est bien vite tombée dans l'oubli, de part et d'autre, hélas! Pourquoi n'a-t-elle pas suscité de nouvelles rencontres à tous les niveaux du dialogue de la vie, des services, de la théologie et de la spiritualité? Il me semble que cela nous oblige à nouveau à nous interroger sur le contenu comme sur les dimensions culturelles et théologiques du dialogue islamo-chrétien. Pour ce faire, une lettre du cardinal Martini, adressée à la population de Milan et intitulée Noi e l'Islam (Nous et l'islam), peut encore nous être utile (section 4). Bien qu'elle date du 6 décembre 1990, elle est étonnamment actuelle. Qu'en est-il advenu? Les choses auraientelles changé depuis lors? Quels en ont été les fruits? Je terminerai ce tour d'horizon en rappelant deux exigences du dialogue qui, pour paraître des truismes, ne sont pas si faciles à mettre en œuvre pour autant: les chrétiens doivent se préparer à répondre adéquatement aux questions posées par leurs interlocuteurs musulmans (section 5) et ils doivent demeurer réalistes sur les écueils qui les attendent dans leur tâche (section 6), en évitant tout autant l'irénisme naïf que la polémique stérile, pour conserver un optimisme où se vit et se reçoit la vertu d'espérance - ce don de Dieu qui est le moteur et l'objectif du dialogue —, témoigner avec et témoigner ensemble d'une espérance à l'œuvre.

\section{La situation internationale a bien changé, mais les papes veulent le dialogue encore et toujours}

Il s'avère qu'après les événements du 11 septembre 2001 et les entreprises belliqueuses qui les ont suivis (et «ce n'est pas fini », hélas!), les soupçons, les amalgames et les accusations se sont faits toujours plus nombreux, de part et d'autre de cette Méditerranée qui devrait unir les uns et les autres en un même idéal de justice et de paix, au nom des civilisations qu'elle a vues naître et s'épanouir sur ses rives. On a trop vite oublié que l'année 2001, la première du troisième millénaire, avait été proclamée par les Nations Unies et l'UNESCO «année du dialogue entre les cultures » et que dans son message du $1^{\text {er }}$ janvier de cette même année, pour la Journée mondiale de la paix, le pape Jean Paul II avait donné au monde un texte merveilleux pour un «Dialogue entre les cultures pour une civilisation de l'amour et de la paix». «L'homme en blanc» qu'il demeure à tout jamais pour nous avait rêvé d'un millénaire de paix, de justice et de réconciliation 
pour tous nos frères et toutes nos sœurs en humanité. Homme de dialogue, à temps et contretemps, il avait osé, au cours de l'Année Sainte de l'an 2000, aller au Sinaï et au Caire, en février, puis en mars à Amman et à Jérusalem. Musulmans, juifs et chrétiens l'avaient écouté parler encore de dialogue et de fraternité. Il allait, plus tard, en mai 2001, visiter la Syrie et transmettre à Damas un même message de paix et d'amitié ${ }^{2}$. Après le drame du 11 septembre, le pape avait visité le Kazakhstan et l'Arménie (22-27 septembre 2001) et invité les chrétiens à jeûner pour la paix avec les musulmans, le 14 décembre, à la fin de leur ramadân. Le $1^{\text {er }}$ janvier 2002, il revenait à la charge, en intitulant son message du Nouvel An: «Il n'y a pas de paix sans justice, il n'y a pas de justice sans pardon ». Peu après, il devait rassembler à Assise des représentants de toutes les religions pour y prier pour la paix, le 24 janvier 2002, confiant ensuite à tous les chefs d'État, le 24 février, le Décalogue d'Assise pour la paix. Qu'en ont-ils pensé, après sa mort, le 2 avril 2005, lorsqu'ils se sont tous retrouvés à ses obsèques, sur la place Saint-Pierre de Rome, pour un ultime hommage à ce grand témoin de l'Esprit? Il avait dissuadé certains chefs d'État d'entreprendre l'aventure belliqueuse de 2003, qui devait engendrer la chute du régime de Saddâm Husayn à Bagdad et entraîner les populations irakiennes dans des années de souffrances et de malheurs. On sait combien il en avait été personnellement affecté.

Le 19 avril 2005, le cardinal J. Ratzinger, qui avait été son étroit collaborateur à la congrégation de la doctrine de la foi, devenait son successeur sous le nom de Benoît XVI et allait poursuivre son «ouverture aux musulmans » en esprit de dialogue, tout en insistant sur la cohérence de la foi chrétienne, en tous ses engagements, et sur la relation étroite qui existe entre religion et culture. C'est dans cette perspective, semble-t-il, qu'il décida, le 15 février 2006, d'unir la présidence du Conseil pontifical pour le dialogue interreligieux (CPDI) et celle du Conseil pontifical pour la culture (CPC), les confiant toutes deux ad interim au cardinal Paul Poupard, président du CPC, et nommant $\mathrm{M}^{\mathrm{gr}}$ M.L. Fitzgerald, jusqu'alors président du CPDI, nonce apostolique au Caire, auprès du gouvernement égyptien, et représentant du Saint-Siège auprès de la Ligue des États arabes. On sait

2. Dans son discours donné dans la cour de la mosquée des Omeyyades, le pape affirmait que "les églises, les synagogues et les mosquées sont des oasis de silence et de prière ". Ce qui a inspiré son titre à la revue semestrielle Oasis qui prit naissance à Venise, en 2005, sur initiative du cardinal Scola. En édition bilingue, franco-arabe, anglo-arabe, italo-arabe, de grand format, elle entend fournir une tribune de dialogue aux intellectuels musulmans et chrétiens de toutes sensibilités. 
que, quelques mois plus tard, le 12 septembre 2006, Benoît XVI prononça à l'Université de Ratisbonne la conférence qui devait paradoxalement donner au dialogue islamo-chrétien un regain d'expressions contrastées. Le $1^{\text {er }}$ septembre 2007, le cardinal Jean-Louis Tauran devenait président du CPDI, redevenu autonome, et le futur cardinal Ravasi, président du CPC. Benoît XVI n'a pas manqué, depuis lors, d'insister sur l'importance du dialogue entre catholiques et musulmans, développant ainsi ce qu'il avait dit à ses hôtes de Castel Gandolfo, le 25 septembre 2006:

Dans un monde marqué par le relativisme et excluant trop souvent la transcendance de l'universalité de la raison, nous avons impérativement besoin d'un dialogue authentique entre les religions et les cultures, capable de nous aider à surmonter ensemble toutes les tensions, dans un esprit de collaboration fructueuse. Poursuivant l'œuvre entreprise par mon prédécesseur, le pape Jean Paul II, je souhaite donc vivement que les relations confiantes qui se sont développées entre chrétiens et musulmans depuis de nombreuses années, non seulement se poursuivent, mais se développent dans un esprit de dialogue sincère et respectueux, fondé sur une connaissance réciproque toujours plus vraie qui, avec joie, reconnaît les valeurs religieuses que nous avons en commun et qui, avec loyauté, respecte les différences.

Ayant repris à son compte la tradition des rassemblements d'Assise, Benoît XVI a pu confirmer, avec succès, son souci du dialogue, lors de son pèlerinage en Terre Sainte, du 8 au 15 mai 2009, que ce soit en Jordanie, en Palestine ou en Israël ${ }^{3}$. Pour résumer l'essentiel de son message, il y a le petit papier qu'il a déposé, à Jérusalem, dans une fente du mur occidental, dit «des lamentations»:

Dieu de tous les âges, au cours de ma visite à Jérusalem, la ville de la paix, la patrie spirituelle des juifs, des chrétiens et des musulmans, je T'apporte les joies, les espoirs et les aspirations, les épreuves, les souffrances et la douleur de tous Tes peuples à travers le monde. Dieu d'Abraham, d'Isaac et de Jacob, entends le cri des affligés, de ceux qui ont peur et de ceux qui souffrent de privations. Envoie Ta paix sur cette Terre sainte, sur le MoyenOrient, sur l'ensemble de la famille humaine et bouleverse les cœurs de tous ceux qui invoquent Ton nom, afin qu'ils marchent humblement sur le chemin de la justice et de la compassion. "Le Seigneur est bon pour ceux qui L'attendent, pour les esprits qui Le cherchent» $(\mathrm{Lm} \mathrm{3,25).}$

3. Tous les textes essentiels de ce pèlerinage se retrouvent dans PISAI (2009). 


\section{Avant et après la conférence de Ratisbonne (12 septembre 2006)}

Les temps ont-ils changé pour autant? Notre monde connaît encore guerres et conflits, catastrophes et misères, divisions et rancœurs. Tout ce qu'il a vécu de drames et de déceptions, ces dernières années, semble confirmer qu' «il n'y a rien de nouveau sous le soleil », comme l'affirmait le Qohelet de la Bible, dans son réalisme pessimiste. Pessimisme et espérance, tels semblent alors être les deux pôles entre lesquels hésitent les hommes et les femmes de dialogue, même si d'autres vont plus loin mettant en doute son efficacité, voire sa légitimité. Depuis la guerre éclair et dévastatrice "du Golfe » (1991), puis les interventions militaires en Irak et en Afghanistan, voici que se sont réveillés les vieux démons de la croisade et du jihâd, que l'on croyait enfin exorcisés, après trente-cinq ans de louables efforts pour un dialogue constructif entre chrétiens et musulmans. Face à la montée des intégrismes, dans les pays à majorité musulmane, et à des manifestations croissantes de xénophobie, dans les pays de tradition chrétienne, beaucoup sont tentés par le doute et le découragement et rejoignent volontiers les rangs des sceptiques ou des contestataires. Le fait est que les opinions publiques sont partout désinformées et désorientées, d'autant plus que les médias semblent prendre un malin plaisir à raviver les soupçons réciproques ou les peurs endémiques. À l'islamophobie des uns, qui voient dans l'islam une religion de violence susceptible d'engendrer le terrorisme, correspond la christianophobie des autres, qui confondent le christianisme avec un Occident dominateur et matérialiste qui aurait perdu son âme. Qui plus est, depuis janvier 2011, les «printemps arabes " sont venus ajouter leur part au cortège des frustrations généralisées et des espérances impossibles: qu'il s'agisse de la Tunisie (toujours en attente d'une constitution), de la Libye (fin dramatique de l'ère Qadhdhâfî), de l'Égypte (divisée en deux camps, le fondamentaliste et le moderniste) et de la Syrie (champ de bataille entre une "armée régulière " et une "armée libre»), sans parler de l'Irak (difficile collaboration entre sunnites, chiites et Kurdes), de l'Afghanistan (insuccès relatif des interventions étrangères), de la Somalie (y naîtra-t-il enfin un État de droit?), du Nigéria (les drames qu'y engendre le mouvement Boko Haram) ou du Mali (les islamistes au pouvoir de sa partie septentrionale), il semble bien que trop nombreux soient les pays musulmans qui se trouvent être en mal de stabilité et d'harmonie! C'est dans ce contexte global que s'insèrent, bon gré mal gré, les initiatives de dialogue et les efforts de collaboration entre chrétiens et musulmans. Ce qui explique les amalgames, les confusions et les polémiques qu'aiment à développer 
ceux et celles qui, de part et d'autre, veulent donner raison au «choc des civilisations» et au «conflit des religions».

Ce contexte permet de comprendre pourquoi la conférence de Ratisbonne a été mal comprise par de nombreux intellectuels musulmans, pourquoi elle a suscité de multiples réactions négatives et ainsi été l'occasion paradoxale d'ouverture d'un débat approfondi sur les possibilités de dialogue islamo-chrétien (voir PISAI 2006; Borrmans 2012, 45-61 et 65-84). Dans son introduction, c'est la citation paradoxale des propos polémiques de Manuel II Paléologue, qui a fait penser que Benoît XVI en assumait personnellement l'attitude apologétique, alors que l'ensemble de la conférence aurait dû plaire aux intellectuels musulmans puisqu'elle était une critique en bonne et due forme de la pensée occidentale positiviste, voire athée. Hélas! Rares sont ceux qui ont lu attentivement et entièrement la susdite conférence. La $1^{\text {re }}$ partie du discours tendait à démontrer que «Dieu ne prend pas plaisir au sang », au nom même de cette raison que tous les croyants ont reçue de Dieu en partage afin d'en harmoniser les exigences avec celles de la foi. La $2^{\mathrm{e}}$ partie de la conférence insistait sur le "rapprochement (historique) entre la foi biblique et le questionnement philosophique grec ", au terme d'un long cheminement qui va de la quête grecque des mystères de l'Être et des interrogations successives de la Bible sur l'être de Yahveh, jusqu'au prologue de l'Évangile selon Saint Jean: "Au commencement était le Verbe, le Logos ", c'est-à-dire "à la fois la raison et la Parole — une raison qui est créatrice et peut se donner en participation, mais précisément comme raison». La $3^{\mathrm{e}}$ partie du discours considérait que «cet intime rapprochement entre la foi biblique et le questionnement philosophique grec est un processus décisif, non seulement du point de vue de l'histoire des religions, mais aussi de celui de l'histoire du monde ». Benoît XVI y voyait aussi «le fondement de ce que, à juste titre, on appelle l'Europe».

Il est certain que la conférence de Ratisbonne n'a pas eu que des effets négatifs, même si les opinions publiques musulmanes en sont restées «choquées ", d'autant plus que, très souvent, elles n'ont guère été informées des explications fournies par Benoît XVI. Il convenait cependant d'en bien connaitre le contenu, car certains penseurs musulmans y ont vu, non sans raison, une critique intelligente d'un rationalisme positiviste occidental, tout comme ils en ont pris occasion pour ouvrir un "dialogue à distance " sur les relations islamo-chrétiennes dans le monde (Borrmans 2007). Ce fut le cas des Réflexions du penseur libanais Ridwân al-Sayyid dans al-Sharq al-Awsat (du 21 septembre 2006), de la Lettre ouverte des 38 'ulamâ' et 
muftî-s de la Fondation Âl al-Bayt de 'Ammân (du 16 octobre 2006)', du Commentaire du musulman libyen Aref Ali Nayed 5 et de la Lettre ouverte $d u$ professeur tunisien Hmida Ennaifer (du 2 octobre 2006) ${ }^{6}$, coprésident du Groupe de recherches islamo-chrétien (GRIC), sans parler du livre intitulé La conférence de Ratisbonne: enjeux et controverses (Bollack, Jambet et al. 2007) ${ }^{7}$. Il n'en reste pas moins vrai que les réactions outrancières manifestées du côté musulman témoignent, à leur manière, des incompréhensions et des préjugés qui demeurent, ainsi que des sensibilités exacerbées que le contexte sociopolitique international n'a fait qu'exciter à la suite des événements du 11 septembre 2001 et des actes de terrorisme et des interventions militaires qui se sont multipliés depuis lors. Les efforts de dialogue entrepris depuis plus de quarante ans auraient-ils été inutiles? On peut légitimement penser que, paradoxalement, la conférence de Ratisbonne en a rappelé l'importance et l'urgence, même si ce n'était pas là l'intention première de Benoît XVI. Celui-ci s'est ainsi vu poussé à s'engager plus que jamais dans le dialogue islamo-chrétien, puisque c'est à l'échelle mondiale que désormais chrétiens et musulmans se doivent d'ap-

4. Cette Lettre des 38 entendait, en ses huit points, préciser, contredire ou approuver la conférence de Ratisbonne: 1. Le "pas de contrainte en religion" est un principe affirmé à Médine et fondamental en islam. 2. Si Dieu est toute transcendance, Il sait aussi être proche de l'homme. 3. L'islam a toujours évité le pur rationalisme et le strict fidéisme. 4. La guerre y a été réglementée, le jihâd est un effort global, les conquêtes islamiques furent de nature politique. 5. L'islam, entité politique, a respecté l'organisation de ses sujets «scripturaires ». 6. Muhammad n'a fait que reprendre les messages antérieurs en les rectifiant. 7. Le recours à des experts exige l'accord de tous. 8. Christianisme et islam se doivent de dialoguer pour la paix mondiale. Les signataires de la Lettre la terminaient d'ailleurs en citant longuement la déclaration conciliaire et les propos de Jean Paul II à Casablanca (1985) et à Rome (1999), tout en remerciant Benoît XVI pour ses mises au point des 17 et 25 septembre. Disponible en ligne: <http:/gric-international.org/2012/approfondir-le-dialogue/textes-musulmans/ lettre-ouverte-de-38-musulmans-a-sa-saintete-le-pape-benoit-xvi/> (consulté le 6 juin 2013).

5. Disponible en anglais en ligne: $<$ http://www.masud.co.uk/ISLAM/misc/commentary _on_benedict.php> (consulté le 6 juin 2013).

6. Disponible en ligne: <http:/gric-international.org/2012/actualite-du-dialogue/lettreouverte-au-pape-lislam-en-partenaire-commentaire-engagement-et-priere> (consulté le 6 juin 2013).

7. Jean Bollack y parle de La Grèce des chrétiens, p. 7-30; Christian Jambet y réfléchit sur Le pape Benoît XVI et l'unité spirituelle de l'Europe (Au temps où l'islam décide de son destin), p. 31-60; Abdelwahab Meddeb y médite sur Le Dieu purifié, p. 61-100. Glucksman, Farouq et al. (2007) étudient la leçon de Ratisbonne, avec les textes de Benoît XVI et les réflexions d'André Glucksman, Wael Farouq, Sari Nusseibeh, Robert Spaemann et Joseph Weiler. 
prendre à «vivre ensemble », dans le respect et l'amitié. Le fait est que, du côté musulman, une lettre importante allait lui être adressée qui engagerait de nouveaux colloques.

\section{La lettre trop vite oubliée des $\mathbf{1 3 8}$ personnalités musulmanes de 'Ammân}

«Une parole commune entre nous et vous ", c'est sous ce titre que cent trente-huit représentants de l'islam contemporain, rassemblés dans le cadre de l'Académie jordanienne de 'Ammân pour les recherches relatives à la civilisation islamique, adressent, le 13 octobre 2007, une lettre ouverte aux chefs religieux des diverses communautés chrétiennes du monde entier, et en premier lieu à Benoît XVI, à l'occasion de la fête de la rupture du jeûne de Ramadân et du premier anniversaire de la Lettre des 38 "savants musulmans» d'octobre 2006. Le titre en était éminemment coranique, puisque les musulmans y sont invités, par leur livre sacré: "Ô Gens du Livre, venez-en à une parole commune entre nous et vous» $(3,64)$. Cette Lettre des 138 se voulait donc l'expression d'un consensus élargi, quant aux signataires, et une reprise de l'un ou l'autre des passages essentiels de la Lettre des 38 de l'année précédente. Sa nouveauté résidait cependant dans une redéfinition du monothéisme qu'affirment, en des formes variées, musulmans, juifs et chrétiens, avec pour thème primordial la même confession du Dieu vivant, un et unique, dans le cadre du double commandement de l'amour de Dieu et du prochain, cher à la tradition judéo-chrétienne. On ne saurait donc trop insister sur «l'esprit d'ouverture» que cette Lettre représentait pour le dialogue islamo-chrétien. C'est pourquoi il convenait d'en comprendre intelligemment la teneur et d'en apprécier positivement les affirmations, non sans s'interroger sur certains de ses silences quant à des versets coraniques qui font encore problème aux chrétiens. Pouvait-on dire qu'elle était représentative de l'islam contemporain? Quelle en était la structure exacte? Dans quelle mesure était-elle innovatrice, tout en se voulant traditionnelle? Comment pouvait-on y discerner une initiative prometteuse d'un dialogue renouvelé? Telles sont les questions qu'il convient de se poser à son sujet et auxquelles une analyse attentive du texte pourrait apporter des réponses justifiées.

Expression d'un "consensus" (ijma') élargi, la Lettre des 138 s'articulait en trois parties: la $1^{\text {re }}$ traitait de l'amour de Dieu (bubb Allâh), en islam et en christianisme; la $2^{\mathrm{e}}$, de l'amour du prochain ( $h u b b$ al-jâr), et la $3^{\mathrm{e}}$ commentait le verset coranique: «Venez-en à une parole commune entre 
nous et vous» $(3,64)^{8}$. Comme en témoigne l'analyse de son contenu, cette Lettre des 138 personnalités musulmanes, qui a suscité le consensus de l'Académie jordanienne Âl al-Bayt et à laquelle ont également adhéré de nombreux autres penseurs de l'islam contemporain, ne pouvait que retenir l'attention des responsables chrétiens du monde entier. Son texte, innovateur et traditionnel tout à la fois, se révélait des plus prometteurs. En effet, pour la première fois, des partenaires musulmans ont voulu aller plus loin dans un dialogue qui s'était trop souvent contenté de la seule plateforme juridique des droits de l'homme et des valeurs qu'ils impliquent. Sans le vouloir, la leçon papale de Ratisbonne avait ainsi amené les uns et les autres à revoir leurs positions, à renouveler leurs réflexions et à susciter de nouvelles initiatives. Préparée par la Lettre des 38, d'expression maladroitement polémique, la Lettre des 138 a alors pu rencontrer un accueil favorable de tous les partenaires chrétiens parce qu'elle les rejoignait dans l'essentiel de leur foi et témoignait d'un effort des musulmans pour mieux interpréter leur monothéisme: le double et unique amour de Dieu et du prochain ne gagnerait-il pas à être médité ensemble, en toutes ses implications théologiques, éthiques et mystiques? À la suite d'échanges de lettres et de visites du prince Ghazi ibn Muhammad ibn Talal, président de l'Âl al-Bayt, deux forums catholico-musulmans ont rassemblé cinquante-huit interlocuteurs des deux côtés, pour en mieux comprendre le contenu, d'abord à Rome (4-6 novembre 2008) (Gaudeul 2008; PISAI 2008) puis en Jordanie (21-23 novembre 2011) (PISAI 2011).

Il faut bien reconnaître, hélas, que si la Lettre des 138 personnalités musulmanes a été largement diffusée dans le monde occidental et a fait l'objet d'un accueil bienveillant de la part des diverses Églises chrétiennes, elle est malheureusement demeurée ignorée des opinions publiques musulmanes, en dehors de la Jordanie et du Liban (Borrmans 2012, 97-98). Bien des faits et des gestes n'ont d'ailleurs guère facilité les choses, étant donné le contexte global des événements internationaux. Certains intellectuels musulmans ont mal accepté le baptême, par le pape, en la nuit de Pâques 2008, du journaliste Magdi Allam qui n'avait eu de cesse de dénoncer le «terrorisme islamique », ainsi que certaines prises de position du Saint-Siège. De son côté, le grand shaykh d'al-Azhar, al-Tayyib, n'a-t-il pas décidé d'interrompre le rythme annuel de la rencontre des délégations chrétienne et musulmane, à la suite des déclarations de Benoît XVI en faveur des

8. Pour mieux connaitre le contenu de la lettre ainsi que sa réception et ses suites, voir Borrmans (2012, 229-247 et 249-268). 
chrétiens du Moyen-Orient, après les massacres de chrétiens, à Bagdad, le 31 octobre 2010 (cathédrale de Notre-Dame du Perpétuel Secours) et à Alexandrie, en Égypte, dans la nuit du Nouvel An 2011 (église des Saints)? Comment interpréter aussi la mise en vigueur par le gouvernement algérien de l'ordonnance présidentielle du 28 février 2006, interdisant tout prosélytisme et réglementant strictement l'exercice de tout culte non musulman? Il est vrai que tout cela n'a pas empêché chrétiens et musulmans de continuer leurs efforts et leurs rencontres. C'est ainsi que le roi d'Arabie Saoudite, Abd Allah bin Abd al-Aziz, a rendu visite au pape à Rome, le 6 novembre 2007 et, après avoir réuni en congrès à La Mecque, sur le dialogue en général, des centaines de 'ulamâ' et de fuqahâ' (4-6 juin 2008), il a organisé le colloque international de Madrid, du 13 au 15 juillet 2008, pour un dialogue entre musulmans, juifs et chrétiens. C’est dans la ligne de ces dernières initiatives qu'a été récemment inauguré, à Vienne, en Autriche, le 26 novembre 2012, non sans dimensions politiques évidentes, le King Abdullah bin Abdulaziz Centre for Interreligious and Intercultural Dialogue (KAICIID). Le Saint-Siège y est représenté en sa qualité d' "observateur fondateur». Il est significatif que, dans toutes ces initiatives, les institutions islamiques ignorent presque totalement ce que font les autres instances musulmanes dans le domaine du dialogue: à La Mecque comme à Madrid et à Vienne, aucune mention ne fut faite de la Lettre des 138 ! Telles sont les limites de tout dialogue interreligieux: il a toujours des dimensions politiques et culturelles, économiques et sociales, tout en essayant d'être plus particulièrement éthique et spirituel.

\section{Actualité de la lettre Noi e l'Islam du cardinal Carlo Maria Martini}

C'est pour la fête de saint Ambroise, le 6 décembre 1990, que le cardinal Martini avait adressé à tous les Milanais une lettre pastorale intitulée Noi e l'Islam ${ }^{9}$. Plus de vingt-deux ans ont passé, mais, à bien la relire, on a l'impression qu'elle date d'aujourd'hui. Après y avoir évoqué les divers aspects de l'intégration des immigrés musulmans, en Italie et spécialement en Lombardie et à Milan, le cardinal posait quatre questions qui sont toujours d'actualité: 1) Que devons-nous penser, nous chrétiens, de l'islam comme religion? 2) L’islam, en Europe, sera-t-il sécularisé, entrant ainsi dans une nouvelle phase de son inculturation en Europe? 3) Quel dialogue

9. Disponible en ligne, en italien : <http://www.chiesadimilano.it/polopoly_fs/ 1.43026.1309792288 !/menu/standard/file/noelislam.pdf> (consulté le 6 juin 2013). 
et, en général, quels rapports, sur le plan religieux, sont-ils possibles entre christianisme et islam en Europe ? 4) L'Église devra-t-elle renoncer à offrir l'Évangile à l'islam?

Pour répondre à la première question, le cardinal commentait les textes de Vatican II relatifs aux musulmans (dans la constitution Lumen Gentium et dans la déclaration Nostra Aetate), ainsi que l'encyclique Redemptoris Hominis de Jean Paul II. Il y constatait que les musulmans vivent d' « une foi qui a de grandes valeurs religieuses et morales ", ce qui les aide à «rendre à Dieu un culte honnête et sincère tout en pratiquant la justice » et donc à "témoigner du primat de Dieu sur toutes choses ». Il n'avait pas à préciser davantage sa vision théologique de l'islam, mais les réflexions et les publications n'ont pas manqué depuis lors, qui tentent de spécifier, du point de vue chrétien, quel peut être la place de l'islam dans l'histoire du salut ${ }^{10}$. Le débat reste entier entre le recueil The Myth of Christian Uniqueness. Toward a Pluralistic Theology of Religions (Hick et Knitter 1987), porte-parole du «relativisme théologique» et le livre Christian Uniqueness Reconsidered. The Myth of a Pluralistic Theology of Religions (D'Costa 1990), porte-parole de "l'inclusivisme d'accueil », qui semble représenter l'attitude la plus juste en la matière, laquelle est aussi celle du Catholic Engagement with World Religions (Becker et Morali 2010). Une position de juste milieu théologique considère qu'il s'agit d'une «religion naturelle d'expression biblique » qui peut inciter le musulman sincère à vivre une foi surnaturelle, grâce à l'intervention de l'Esprit Saint. C'est dans ce sens qu'il conviendrait de relire l'encyclique Redemptoris Missio de Jean Paul II (7 décembre 1990).

Quant à la deuxième question, il est bien difficile d'y répondre, tant les situations sont contrastées et les communautés diversifiées en Europe. Les études et les publications sont nombreuses et chaque pays y connaît un contexte qui lui est spécifique (Branca 2005; Borrmans 2005a). Mais si, dans certains cas, des musulmans se sont fait un islam modernisé et si des institutions de dialogue se sont développées sur le plan national, force est de reconnaître que, dans leur grande majorité, les communautés musulmanes en Europe affirment davantage leur identité et tendent à une plus grande visibilité. Il s'ensuit que les conditions actuelles de dialogue se sont

10. Pour l'histoire de la théologie chrétienne à ce sujet, voir Rizzardi (2005a, 2005b); Chiappo (2005); Crociata (2006); Jukko (2007). 
faites plus difficiles ${ }^{11}$. Il suffit d'évoquer, à titre d'exemple, les faits suivants. En Grande-Bretagne, l'application de la sharî'a, conformément à l'Arbitration Act de 1996, qui régule le domaine de son application à condition que "les décisions prises soient acceptées par les deux parties", fait l'objet d'un vif débat. Car certaines des décisions des tribunaux appliquant la sharî'a (ils sont 83 en 2009) sont "discriminatoires envers les femmes dans des domaines, tels que la garde des enfants, la violence domestique et le divorce ». Or, le nombre des musulmans qui y recourent ne fait qu'augmenter: faut-il donc les défendre ou les supprimer? En Belgique, la cour constitutionnelle rejette les recours contre la loi antiburqa, justifiant ainsi la constitutionnalité de celle-ci, mais un petit parti «islam» a remporté des sièges à des élections municipales ${ }^{12}$. En France, l'article 1 de la loi du 15 mars énonce que, «dans les écoles, les collèges et les lycées publics, le port de signes ou tenues par lesquels les élèves manifestent ostensiblement une appartenance religieuse est interdit», mais le ministre de l'Intérieur, également ministre des Cultes, participe parfois à l'inauguration d'une nouvelle mosquée. L'État reconnaît et finance des aumôneries musulmanes, dans les hôpitaux, les armées et les prisons, et organise les élections aux conseils régionaux du culte musulman ${ }^{13}$. En Allemagne, la communauté Milli Görüs est sur la liste noire de «l'État fédéral pour la protection de la constitution entre le public et les députés et les réformes internes». L'interdiction des mutilations corporelles pose des problèmes à la pratique du rite de la circoncision chez les juifs et les musulmans. Ces quelques exemples disent assez combien l'intégration des musulmans dans les divers pays européens dépend étroitement du type

11. Pour les relations «États et religions» dans l'Europe des 25 membres de l'Union, voir Massignon (2007); pour les législations les concernant, pays par pays, voir Massignon et Riva (2010).

12. On y envisage aussi des cours d'islam pour les élèves musulmans fréquentant les écoles catholiques, tandis que l'armée belge redoute des infiltrations salafistes dans son sein et que les «banques islamiques» se font toujours plus nombreuses dans le pays.

13. Autres faits: l'imâm tunisien Hammami, de le mosquée Omar (Paris, $11^{\mathrm{e}}$ ), du mouvement Foi et Pratique (Tabligh), a été récemment expulsé, car le ministre de l'Intérieur refuse qu'on y tienne des « discours de haine ». Les affiches d'une campagne du collectif contre l'islamophobie n'ont pas été autorisées dans les couloirs du métro de Paris. Les Facultés catholiques de Paris et de Lyon organisent pour des imâms des cours de «connaissance de la laïcité » française. Mais que penser du regain de spécialisation des boucheries et des carrés de supermarchés en produits halâl? 
d'islam qui y est enseigné dans les mosquées ou transmis dans les associations: on ne saurait dire que l'islam s'y soit sécularisé dans son ensemble.

Quant aux troisième et quatrième questions de la lettre du cardinal, les réponses y sont nombreuses, car les efforts de dialogue se sont multipliés en Europe, au cours des dernières années, à la suite des textes du CPDI du 10 mai 1984: Attitude de l'Église catholique devant les croyants des autres religions, et du 19 mai 1991: Dialogue et annonce: réflexions et orientations concernant le Dialogue interreligieux et l'Annonce de l'Évangile, du document: Le christianisme et les religions de la Commission théologique internationale (octobre 1996) et de celui de la congrégation pour la doctrine de la foi, du 6 août 2000: Sur l'unicité et l'universalité salvifique de Jésus-Christ et de l'Église, sans parler des lettres pastorales d'évêques ou de conférences épiscopales ${ }^{14}$. Qu'il suffise de consulter ici les revues Islamochristiana (Rome), Chemins de dialogue (Marseille) et Oasis (Venise), pour s'en faire une idée. À titre d'exemple, la lettre du secrétariat pour les relations avec l'islam (SRI) de Paris et le bulletin mensuel parisien Se Comprendre donnent toutes les informations nécessaires sur les formes du dialogue en France. Le comité «Islam en Europe », de l'assemblée conjointe du conseil des conférences épiscopales européennes (CCEE) (catholique) et de la conférence européenne des Églises (KEK) (œcuménique) n'a pas manqué d'inspirer des rencontres de dialogue, telle la toute dernière, celle de Sarajevo (5 décembre 2012). Dans sa Declaration of European Religious Leaders, en faveur d'une "société saine", il présente sept options pour rendre efficace le «vivre ensemble»: respect de la diversité vécue dans l'interdépendance, respect des valeurs spirituelles, compassion, souci de la liberté et des droits démocratiques, créativité et esprit critique. Ce comité a également publié: La présence des musulmans en Europe et la formation théologique des agents pastoraux (1991), Réciprocité islamo-chrétienne: éléments de réflexion pour les Églises européennes (1995), Mariages entre

14. S'agissant plus particulièrement de la France, il y a la déclaration commune des évêques réunis à Lourdes: Catholiques et musulmans: un chemin de rencontre et de dialogue (1998), précédée par la lettre pastorale de $\mathrm{M}^{\mathrm{gr}}$ Pierre Raffin: Visages de l'islam et regard chrétien, jalons pour le dialogue islamo-chrétien en Moselle (1993) et suivie de la note de la commission doctrinale des évêques de France intitulée: Comment chrétiens et musulmans parlent-ils de Dieu? (2008) - textes disponibles en ligne: <http://www.cdo-lyon.cef.fr/spip.php ?rubrique73> (consulté le 6 juin 2013). À signaler aussi le dernier document du conseil pour les relations interreligieuses des évêques de France: Fondements et objectifs $d u$ dialogue interreligieux (2009), ainsi que les hors-série de La Documentation Catholique de Paris: L'Église et l'Islam (1995) et Questions actuelles: l'Église et l'Islam en France (2000). 
chrétiens et musulmans: orientations pour les Églises et les chrétiens en Europe (1997), Aller à la rencontre des musulmans (2003) et Chrétiens et musulmans: citoyens d'Europe et hommes de foi (2008).

\section{Aider les chrétiens à bien répondre aux questions qu'on leur pose}

Le cardinal Martini achevait sa lettre en indiquant la «position correcte» pour les chrétiens: ni indifférentisme ni prosélytisme, mais bien plutôt « un effort sérieux de connaissance » pour mieux comprendre et, par la suite, témoigner de sa propre foi en tenant compte de la sensibilité des musulmans. Sans renoncer à offrir l'Évangile à ces derniers, il faut toujours bien distinguer dialogue et annonce. Celle-ci est "la proposition simple et désarmée de ce qui nous apparaît de plus cher [...] du mystère de Dieu même» révélé en Jésus Christ par "le témoignage quotidien de la charité et du don de soi-même aux autres ». Mais le dialogue vise à "l'émulation spirituelle » entre les partenaires qui s'estiment réciproquement, d'un respect têtu, en vue d'être meilleurs dans leur propre tradition et de mieux servir leurs frères en humanité. Et le cardinal d'évoquer alors l'exemple de la communauté chrétienne de Milan, au temps de saint Ambroise. Il est certain qu'on ne peut plus catéchiser les enfants aujourd'hui sans leur proposer un regard chrétien sur les religions non chrétiennes, tout comme les prédications et les méditations en Église se doivent de faire place à une attitude d'accueil vis-à-vis de l'expérience religieuse des non-chrétiens. Les publications ne manquent pas pour informer honnêtement et former chrétiennement ceux et celles qui ont à dialoguer avec les musulmans. C'est pourquoi j'ai édité jadis, au nom du secrétariat pour les non-chrétiens, des Orientations pour un dialogue entre chrétiens et musulmans (Borrmans 1981), repris plus tard sous la forme d'un livret: Pour comprendre les Musulmans (Borrmans 2010). Pour mieux préparer les chrétiens à justifier «l'espérance qui est en eux ", des livres plus adaptés ne manquent pas, tel celui du père Robert Caspar (1987): Comment répondre aux questions qu'on nous pose? ${ }^{15}$, et celui du père Christian Troll (2011): Que répondre aux musulmans?

Comme le faisait penser le cardinal Martini, dans sa lettre de 1990, les musulmans ne sont pas insensibles aux valeurs de l'Évangile et ils se posent

15. En voici les thèmes essentiels: le prophétisme de Muhammad, le célibat consacré, les motifs de notre présence, unicité de Dieu et Trinité, la messe et l'eucharistie, la divinité de Jésus et l'incarnation, le péché originel et la croix (la rédemption), les Écritures et la parole de Dieu, spirituel et temporel, l'Église, la prière, la pluralité des religions et la liberté religieuse, qu'est-ce que le christianisme? 
parfois des questions sur l'enseignement de ce Jésus, fils de Marie, dont ils savent qu'il fut un grand prophète et dont la mère, Marie, fille de 'Imrân, était virginale. J'ai eu l'occasion d'en évoquer l'importance, dans une conférence donnée à l'Université Urbaniana, en octobre 2005 (Borrmans 2009). Les musulmans disent croire à l'Évangile: il serait possible d'en parler mieux avec eux, en tenant compte de leurs objections, car les Béatitudes leur sont acceptables, voire désirables. Si Jésus est connu par eux comme Messie thaumaturge ou Envoyé de Dieu, il est donc possible d'en parler comme d'un maître de sagesse et d'un guide spirituel. Les chrétiens devraient savoir ce qu'en disent le Coran et la Tradition, et expliquer ensuite, à partir des expressions musulmanes et en les développant, ce qu'il en est finalement de ce Jésus et de Marie, sa mère. Pour cela, tout responsable de la préparation des chrétiens qui auront à s'expliquer sur Jésus Christ devrait consulter les livres essentiels sur celui-ci afin d'accompagner les musulmans dans leurs interrogations sur son identité (Arnaldez 1980 et 1988; Borrmans 2005b; Khalidi 2003). L'essentiel, puisqu'ils mettent en doute l'authenticité de nos évangiles canoniques, n'est-il pas de mettre sous leurs yeux le "cinquième évangile» que devrait être tout chrétien qui vit sa foi avec cohérence et devient alors un "évangéliste » en paroles et en actes? Ce faisant, il est possible de mieux comprendre les exigences du véritable dialogue du côté chrétien: d'une part, devenir "comme l'hôte» de l'autre, familier de sa tradition spirituelle et habitué à son vocabulaire religieux, et, d'autre part, se sentir "configuré au Christ» en son mystère d'incarnation et de rédemption (nié par le Coran), tout en vivant une spiritualité de compassion et de substitution, comme y invitait Louis Massignon, un grand témoin de ce dialogue (Massignon, Borrmans et al. 2011).

Puisque le cardinal Martini invitait les chrétiens à ne pas renoncer à " offrir l'Évangile» aux musulmans, encore faudrait-il bien s'entendre sur ce sujet. Si certains de leurs frères chrétiens, appelés "évangéliques", se révèlent souvent trop pressés d'offrir un exemplaire de la Bible et de proposer au musulman d'adhérer à Jésus Christ, au risque d'être accusés de prosélytisme, les catholiques risquent plutôt de pécher par un excès de réserve et par un silence timide. Or, il leur faut bien rendre compte de leur propre foi, quand ils sont interrogés sur celle-ci, et expliquer aux musulmans toutes les valeurs chrétiennes qui sont impliquées dans la culture européenne, à laquelle ils ont décidé de s'intégrer. Si les fidèles de l'islam savent que toute conversion à Jésus Christ est un délit et un crime, selon la sharî 'a, en terre d'islam, ils doivent aussi savoir qu'il n'en est pas de même, en Europe, où toute liberté religieuse est garantie à tous. Les chrétiens ont 
le devoir de répondre à toutes leurs questions en matière religieuse, confessant ainsi leur foi et invitant les musulmans à vivre la leur, "en esprit et en vérité ». Dans le cadre de «l'année de la foi» (11 octobre 2012-24 novembre 2013) et de la "nouvelle évangélisation ", les catholiques doivent se garder de tout péché d'omission en ce domaine. Bien des musulmans ne comprendraient pas qu'ils restent silencieux, quand il s'agit des questions que se pose tout homme bien né: Qu'est-ce que la vie? Qu'est-ce que la mort? D'où venons-nous et où allons-nous? Quel est le sens de la souffrance? Quelle est la réalité suprême qui se cache au plus profond du cœur de l'homme? Les meilleurs des chrétiens pourraient alors bénéficier des confidences de ceux qui ont «accompli » leur «islam spirituel», en se convertissant à Jésus Christ et en entrant dans son Église, tels $\mathrm{M}^{\text {gr }} \mathrm{Paul}$ Méhémet-Ali Mulla-Zadé (Mula-Zadé et Molette 1988), le père JeanMohamed Abd-el-Jalil (Abd-el-Jalil et Borrmans 2004; Mulla-Zadé, Abdel-Jalil et al. 2009) et le père Afîf Osseïrane (Keryell 2009).

\section{Les difficultés réelles du dialogue}

Contesté par les fondamentalistes chrétiens et musulmans de tous bords ou volontiers pratiqué par les humanistes culturels des deux traditions religieuses, le dialogue risque trop souvent d'être réduit à d'excellentes relations humaines qui tendent à négocier en commun les règles d'un "vivre ensemble» le plus harmonieux possible. On risque alors d'oublier l'importance religieuse et surtout les enjeux spirituels d'un tel dialogue, tout en feignant d'en ignorer les difficultés, les règles et les limites. Car le dialogue interreligieux n'a pas pour but d'organiser un vague æcuménisme où les religions se verraient également reconnues comme autant de voies égales de salut pour leurs adhérents ou adeptes. Il est évident que, s'agissant du dialogue islamo-chrétien, les responsables chrétiens et musulmans ne l'envisagent pas de la même manière. Si, du côté chrétien, les textes ne manquent pas, qu'ils relèvent de l'Église catholique ou du conseil œcuménique des Églises, il n'en est pas de même du côté musulman : trop souvent, les intellectuels qui le pratiquent n'engagent que leur responsabilité personnelle, tandis que les institutions peinent à en définir les modalités ou ne l'envisagent que comme une forme de da'wa (faire connaître l'islam «mal connu », sinon vitupéré, et faire respecter le Prophète de l'islam, trop souvent dénigré). Il n'en reste pas moins vrai que la difficulté principale est inconsciemment d'ordre théologique: tout musulman est convaincu, de par 
le Coran lui-même, que le christianisme des chrétiens est un faux christianisme (une espèce d'hérésie paulinienne). Pour lui, le véritable christianisme est celui que lui enseigne son livre, le Coran, à savoir un simple monothéisme semblable à celui de l'islam, dont Jésus est le prophète, transmetteur d'un livre (l'évangile) et annonciateur de Mahomet/Muhammad. Le chrétien souffre alors de voir son monothéisme soupçonné de polythéisme, ses livres saints accusés de falsification et le mystère de la croix officiellement nié historiquement. Heureux sera-t-il quand il saura faire admettre au partenaire musulman, phénoménologiquement parlant, que les valeurs supérieures que celui-ci admire en lui proviennent directement du christianisme, tel qu'il est transmis par vingt siècles de fidélité réfléchie et de sainteté vécue. Le fait est que les versets coraniques de reproches faits aux chrétiens sont habituellement interprétés comme dénonçant les mystères de la Trinité, de l'incarnation et de la rédemption. C'est de là que découlent tant de préjugés que presque tous les musulmans expriment vis-à-vis de la foi, de la morale et des rites des chrétiens. C'est de là aussi que dérivent les discriminations juridiques engendrées par la "différence de religion" (la musulmane ne peut pas épouser un chrétien et aucune vocation successorale n'existe entre chrétiens et musulmans).

C'est de là que proviennent donc les nombreuses difficultés «pratiques» de la vie quotidienne, qu'il s'agisse des prescriptions alimentaires, des traditions vestimentaires et des relations sociales les plus élémentaires. Que peut faire, en effet, un directeur d'école ou un président d'association devant le refus de toute mixité, même à table, lors de repas de convivialité amicale? Comment devrait réagir celui à qui est refusée une poignée de main de politesse courtoise? Que dire à celui qui refuse les plats de l'hospitalité généreuse parce que la viande n'a pas été préparée "à la musulmane» ou parce qu'à table il y a des bouteilles de vin pour les chrétiens? Ce ne sont là que quelques-unes des difficultés auxquelles se heurte le dialogue de la vie quotidienne et c'est malheureusement sur ces détails qu'insiste l'affirmation identitaire de certains, encouragée par des prédicateurs réticents à toute adaptation à la modernité ou au pluralisme. La volonté qu'ont certains de marquer sociologiquement la différence ne facilite donc pas le "vivre ensemble", dans les écoles, les associations et les familles mixtes. Il est certain qu'une meilleure lecture de certains versets coraniques, ou de nombreuses traditions qui n'en relèvent pas directement, pourrait jouer en faveur du dialogue lui-même. Or, une telle lecture relève des responsables de l'institution religieuse elle-même et ces derniers ne sont guère préparés à oser «l'aventure du dialogue ». D'où l'importance incon- 
tournable des rencontres entre ceux qui ont à orienter les opinions publiques vers une meilleure "présence à l'autre». Quels sont les exégètes ou les théologiens musulmans qui auront le courage de "contextualiser" les versets de violence du Coran, comme le suggèrent certains musulmans libéraux? Cela s'avère d'autant plus nécessaire que la montée des islamophobies et des christianophobies est toujours plus grande, à cause du contexte politique international: de chaque côté, les amalgames ne sont que trop faciles, qui confondent islam et terrorisme ou christianisme et Occident. Pour compliquer les choses, il y a l'étrange volonté des médias à ne transmettre que ce qui a caractère conflictuel dans la relation entre chrétiens et musulmans, et le silence inexplicable de trop nombreux responsables religieux de l'islam qui ne dénoncent pas urbi et orbi les méfaits indûment commis au nom de celui-ci. C'est pourquoi, face à toutes ces difficultés, le dialogue se doit d'être cohérent avec son esprit et réaliste avec les faits, généreux dans sa pratique et respectueux de ses limites, persévérant dans son idéal et patient dans la réalisation de celui-ci.

\section{Conclusion}

Il fallait bien évoquer toutes ces dimensions du dialogue islamo-chrétien tel qu'il se présente aujourd'hui. Trop nombreux sont ceux qui, du côté chrétien comme du côté musulman, le jugent inutile ou dangereux, au risque de donner raison à la thèse de Samuel Huntington qui ne voit d'avenir que dans un "choc des civilisations" religieuses. Le présent article a voulu offrir des informations et des réflexions en vue d'une meilleure compréhension des difficultés actuelles, des contextes culturels et des exigences spirituelles du dialogue islamo-chrétien. Le pape Benoît XVI l'a affirmé à Rome, le 25 septembre 2006: «Le dialogue interreligieux entre chrétiens et musulmans [...] est une nécessité vitale, dont dépend, en grande partie, notre avenir ${ }^{16}$.» La situation internationale connaît des changements toujours plus inattendus, si bien que les papes veulent voir les chrétiens dialoguer toujours plus avec les croyants des grandes religions mondiales, et l'islam en premier lieu. La conférence de Ratisbonne a eu le mérite d'attirer l'attention sur les dimensions inéluctablement intellectuelles et théologiques du dialogue, ce que ses promoteurs ont toujours

16. Discours disponible en ligne: <http://www.vatican.va/holy_father/benedict_xvi/ speeches/2006/september/documents/hf_ben-xvi_spe_20060925_ambasciatori-paesiarabi_fr.html> (consulté le 6 juin 2006). 
considéré comme essentiel, dans une recherche commune de la vérité. Qui plus est, la Lettre des 138 personnalités musulmanes de 2007 devrait constituer une plateforme permanente de dialogue théologique entre chrétiens et musulmans, car elle engage leur commun service des droits de l'homme. Mais c'est la lettre du cardinal Martini de 1990 qui devrait inciter les chrétiens à mieux répondre aujourd'hui aux quatre interrogations qu'il soumettait aux diocésains de Milan. Les chrétiens sont-ils prêts à rendre compte de leur foi d'une manière qui soit compréhensible pour les musulmans? C'est ici qu'il convient de faire un examen de conscience: comment y sont-ils préparés? Les efforts en ce sens n'ont pas manqué, depuis Vatican II, mais ils se sont avérés insuffisants. Puisque les musulmans se sont faits plus nombreux en Europe, les chrétiens se doivent donc d'être parfaitement éduqués au dialogue interreligieux, instruits des connaissances qu'il suppose et initiés à la spiritualité qu'il exige.

\section{Références}

AbD-el-Jalil, J.-M. et Borrmans, M. (2004), Jean-Mohammed Abd-elJalil, témoin du Coran et de l'Évangile. De la rupture à la rencontre, Paris, Cerf / Éd. franciscaines (L'histoire à vif).

Arnaldez, R. (1980), Jésus, fils de Marie, prophète de l'islam, Paris, Desclée (Jésus et Jésus-Christ 13).

(1988), Jésus dans la pensée musulmane, préf. par Joseph Doré, Paris, Desclée (Jésus et Jésus-Christ 32).

Becker, K. J., Morali, I. et al. (2010), Catholic Engagement with World Religions: A Comprehensive Study, Maryknoll, Orbis Books (Faith meets faith).

Bollack, J., Jambet, C. et al. (2007), La Conférence de Ratisbonne, Paris, Bayard.

Borrmans, M. (2005a), «Comunità musulmane nell'Europa occidentale », Ad Gentes, 9/2, p. 133-138.

- $\left(2005 b^{2}\right)$ [1996], Jésus et les musulmans d'aujourd'bui, Paris, Desclée (Jésus et Jésus-Christ 69).

(2007), «Reazioni musulmane alla conferenza di Ratisbona. Polemica o dialogo ? », Ad Gentes, 11/2, p. 159-183.

(2009), «Un "Évangile" disponible à tous les musulmans ", Rivista Espanola de Teologia, 69, p. 205-226. 
(2009), Prophètes du dialogue islamo-chrétien: Louis Massignon, Jean-Mohammed Abd-el-Jalil, Louis Gardet, Georges C. Anawati, Paris, Cerf.

(2010), Pour comprendre les musulmans, Paris, Médiaspaul.

(2012), Dialoguer avec les musulmans. Une cause perdue ou une cause à gagner?, préface du cardinal Jean-Louis Tauran, Paris, P. Téqui (Questions disputées).

(2013), "Dialogare con i musulmani, ancora e sempre», Ad Gentes, 17/1, p. 52-66.

(à paraître), Témoins du dialogue islamo-chrétien: Roger Arnaldez, Robert Caspar, Jacques Jomier, Youakim Moubarac, Paris, Cerf.

Borrmans, M. (1981), dir., Orientations pour un dialogue entre chrétiens et musulmans, Secrétariat pour les non-chrétiens, Paris, Cerf.

(2002), Dialogue islamo-chrétien à temps et contretemps / avec la collab. éditoriale d'Annie Laurent, Versailles, Éd. Saint-Paul.

BrancA, P. (2005), «I musulmani e la società civile », Ad Gentes, 9/2, p. 139152.

Caspar, R. (1987), Traité de théologie musulmane, tome I: Histoire de la pensée religieuse musulmane, Rome, PISAI.

(1988), Comment répondre aux questions qu'on nous pose?, Rome, PISAI.

- (2006), Pour un regard chrétien sur l'islam, Paris, Bayard.

Chiappo, M. (2005), "L'islam nel magistero delle Chiese europee ", Ad Gentes, 9/2, p. 204-224.

Crociata, M. (2006), dir., Per un discernimento cristiano sull'islam, Rome, Città Nuova.

D’Costa, G. (1990), Christian uniqueness reconsidered: The Myth of a Pluralistic Theology of Religions, Maryknoll, Orbis Books (Faith meets faith).

Gallez, É.-M. et CatTenoz, J.-P. (2012), Le malentendu islamo-chrétien I postf. de $\mathrm{M}^{\mathrm{gr}} \mathrm{J}$.-P. Cattenoz, Paris, Salvator.

Gaudeul, J.-M. (2008), «Rome: le Forum islamo-catholique», Se Comprendre, 8/10, <http://www.comprendre.org/08_10.pdf >, (consulté le 31 mai 2013), 
Glucksman, A. , Farouq, W. et al. (2007), Dio salvi la ragione, Siena, Cantagalli.

Hick, J. et Knitter, P. F. (1987), The Myth of Christian Uniqueness: Toward a Pluralistic Theology of Religions, Maryknoll, Orbis Books (Faith meets faith).

Jukкo, R. (2007), «Fondamenti teologici del Pontificio Consiglio per il Dialogo Interreligioso nelle sue relazioni con i musulmani », Ad Gentes, 11/2, p. 145-158.

Keryell, J. (2009), Afif Osseïrane, un chemin de vie, Paris, Cerf.

KHALIdI, T. (2003) [anglais 2001], Un musulman nommé Jésus, dits et récits dans la littérature islamique, trad. de l'anglais et de l'arabe pour le texte des dits et récits par Jean-Louis Bour, Paris, Albin Michel (L'islam des Lumières).

Massignon, B. (2007), Des dieux et des fonctionnaires, religions et laïcités face au défi de la construction européenne, préf. de J.-P. Willaime, Rennes, Presses universitaires de Rennes (Sciences des religions).

Massignon, B., Riva, V. et al. (2010), L'Europe, avec ou sans Dieu, héritages et nouveaux défis, Institut européen en sciences des religions (IESR-EPHE), Ivry-sur-Seine/Namur/Lausanne, Éd. de l'Atelier/Fidélité/ Éd. D'En-bas.

Massignon, L. (2011), Badaliya, au nom de l'autre, 1947-1962, présenté et annoté par Maurice Borrmans et Françoise Jacquin, préface du cardinal Jean-Louis Tauran, Paris, Cerf (Patrimoines Islam).

Mulla-Zadé, P. Mehemet-Ali, Abd-el-Jalil, J.-M. et al. (2009), Deux frères en conversion du Coran à Jésus. Correspondance, 1927-1957, rassemblée, introduite et annotée par Maurice Borrmans, Paris, Cerf (Intimité du christianisme).

Mulla-Zadé, P. Mehemet-Ali et Molette, C. (1988), Mulla - Une conscience d'homme dans la lumière de Maurice Blondel, textes réunis et publiés par C. Molette, Paris, Téqui.

PISAI, Pontificio Istituto di Studi Arabi e d'Islamistica (2006), «Dossier "Regensburg" ", Islamochristiana, 32, p. 273-297.

(2008), "Love of God, Love of Neighbour: First Seminar of the Catholic-Muslim Forum ", Islamochristiana, 34, p. 261-272.

(2009), «Pilgrimage of His Holiness Benedict XVI to the Holy Land ", Islamochristiana, 35, p. 254-267. 
(2011), "Catholic-Muslim Forum: Second Seminar », Islamochristiana, 37, p. 264-285.

Poggi, V. et Borrmans, M. (2012), Paul Ali Mehmet Mulla-Zadé, islamologo di tre papi-Lettres de Mulla-Zadé à Louis Massignon, Rome, PIO.

RizzARDi, G. (2005a), "L'interrogazione teologica cristiana e la religione islamica nei secoli VIII-XIV», Ad Gentes, 9/2, p. 153-176. (2005b), «La teologia nel nuovo contesto dell'islamologia cristiana nei secoli XCIX-XX », Ad Gentes, 9/2 (no 18), p. 177-203.

Troll, C. W. (2011), Que répondre aux musulmans?, trad. de l'allemand par J. Weisshaupt, Namur, Fidélité.

\section{Résumé}

Les Églises locales, en Europe, ont multiplié leurs institutions pour dialoguer avec les musulmans immigrés pour en développer toutes les possibilités: à côté de leurs fidèles engagés dans ce dialogue, beaucoup d'autres le critiquent ou le refusent pour mille raisons politiques ou culturelles, voire théologiques et spirituelles. Les papes Jean Paul II et Benoît XVI insistent sur la nécessité de le poursuivre «encore et toujours" au nom même de l'Évangile, malgré les difficultés du moment. Même l'incident de parcours que fut la conférence de Ratisbonne (12 septembre 2006) s'est révélé être une incitation à en approfondir les modalités et les limites. Quant à la Lettre des 138 personnalités musulmanes d'octobre 2007, riche en promesses, elle a été trop vite oubliée de part et d'autre. La Lettre du Cardinal Martini au peuple de Milan, de décembre 1990, est pourtant toujours d'actualité. Les chrétiens sont invités à rendre compte de leur foi, à bien connaître les musulmans et à leur faire découvrir les valeurs chrétiennes de la culture européenne. Tous doivent être réalistes dans leurs collaborations en y tenant compte des difficultés qu'il y a à «vivre ensemble» quand on appartient à des cultures différenciées: que chacun y fasse effort pour être plus proche de l'autre.

\section{Abstract}

Local Churches in Europe have multiplied institutions to develop all possible options of dialogue with Muslims immigrants. If some of the Church members are committed to the dialogue, many other ones are opposed to it for political, cultural, and even theological and spiritual reasons. Popes John Paul II and Benedict XVI insist on the necessity to pursue the dialogue again 
and again in the name of the Gospel, even during difficult times. Even the stumbling stone that the Regensburg conference has been (September 12, 2006) turned out to be an occasion to deepen the dialogue's means and limits. The promising Letter written by $138 \mathrm{Muslim}$ representatives in October 2007 has been too quickly forgotten from both sides but the Letter form cardinal Martini to Milan in December 1990 is still relevant. Christians are invited to testify of their own faith, to know Muslims better, and to spread the Christian values of European culture. Everyone need to be realistic as they collaborate, taking into account how hard it is to live together when you belong to different cultures: everyone need to make their own efforts to get closer to each other. 